DOI: http://doi.org/10.22585/hospdomic.v5i2.130

\title{
Abordaje de la hiperpotasemia: desde urgencias a la unidad de hospitalización domicilio
}

\section{Management of hyperkalemia: from the emergency department to the home hospitalization unit}

\author{
Álvaro Fuentes Merlos' (1) 0000-0002-1024-1620 \\ Francisco Garrido Navarro² (D) 0000-0002-1370-3421 \\ Rafael López-Bas Valero³ (D) 0000-0003-2006-9243 \\ 1. Hospital Universitario de San Juan de Alicante, Servicio de Urgencias Hospitalarias, Sant Joan d'Alacant, España \\ 2. Hospital Universitario de San Juan de Alicante, Unidad de Hospitalización a Domicilio, Sant Joan d'Alacant, España.
}

Correspondencia/Correspondence

Álvaro Fuentes Merlos

afuentesmerlos@gmail.com

Recibido/Received

31.01 .2021

Aceptado/Accepted

24.02.2021

Conflicto de Intereses/Competing interest

Los autores declaran que no existe conflicto de interés alguno en

el presente trabajo.

\section{Financiación/Funding}

Este trabajo no ha requerido financiación alguna.

Contribuciones de autoría/Author contributions

Todos los autores han contribuido por igual en la realización de este trabajo.

\section{CÓMO CITAR ESTE TRABAJO | HOW TO CITE THIS PAPER}

Fuentes A, Garrido F, López-Bas R. Abordaje de la hiperpotasemia: desde urgencias a la unidad de hospitalización domicilio. Hosp Domic. 2021 ; 5(2): 125-30. 


\section{RESUMEN}

La hiperpotasemia es una anomalía eléctrica común que puede empeorar arritmias cardíacas y aumentar significativamente la mortalidad. El envejecimiento progresivo de la población sumado a la mayor incidencia de entidades como la diabetes mellitus (DM), enfermedades cardiovasculares (ECV) o enfermedad renal crónica (ECV) así como el creciente uso de inhibidores del sistema renina angiotensina aldosterona (RAASi) ha provocado que sea un problema frecuente al que tenga que enfrentarse el clínico en su día a día. Presentamos un caso de un paciente ingresado a cargo de la Unidad de Hospitalización a Domicilio de San Juan de Alicante que presentaba un cuadro de inicio súbito y clínica inespecífica debido a una hiperpotasemia grave. Tras estabilización previa en el servicio de urgencias, se realizó una combinación de tratamiento con fármacos RAASi, diuréticos de asa y resinas de intercambio catiónico (patiromero) presentando una mejoría de la sintomatología y control de la potasemia.

Palabras clave: Hiperpotasemia; Terapéutica; Sistema Renina-Angiotensina

\section{ABSTRACT}

Hyperkalemia is a common electrical abnormality that can worsen cardiac arrhythmias and significantly increase mortality. The progressive aging of the population added to the higher incidence of entities such as diabetes mellitus (DM), cardiovascular diseases (CVD) or chronic kidney disease (CVD) as well as the increasing use of renin angiotensin aldosterone system inhibitors (RAASi) has caused make it a frequent problem that clinicians have to face in their day-to-day life. We present a case of a patient admitted to the San Juan de Alicante Home Hospitalization Unit who presented a sudden onset and nonspecific clinical picture due to severe hyperkalemia. After prior stabilization in the emergency department, a combination of treatment with RAASi drugs, loop diuretics and cation exchange resins (patiromer) was performed, presenting an improvement in symptoms and control of potassium levels.

Keywords: Hyperkalemia; Therapeutics, Renin-Angiotensin System 


\section{INTRODUCCIÓN}

La hiperpotasemia es un trastorno electrolítico común, especialmente en enfermedad renal crónica (ERC), diabetes mellitus (DM) o enfermedad cardiovascular (ECV), reportándose una prevalencia de hasta $10 \%$ en pacientes hospitalizados ${ }^{(1)}$ recomienda la estratificación de la hiperpotasemia en leve (5,5-5,9 mmol/L), moderada (6,0-6,4 mmol/L) y grave (>6,5 mmol/L) para ayudar a la toma de decisiones clínicas ${ }^{(5)}$. El tratamiento se basa en una estrategia de tres pasos, a saber, la cardioprotección, el desplazamiento celular de potasio y la eliminación de potasio.

Los factores predisponentes para la hiperpotasemia implican un aumento de la ingesta/carga de potasio, una redistribución celular anormal, el empeoramiento de la capacidad ya disminuida de excretar el potasio en pacientes con ERC, o a menudo una combinación de estos factores. Otras dos comorbilidades que causan directa o indirectamente hiperpotasemia son la DM y las ECV (2). Entre los medicamentos, los bloqueadores de los receptores beta-2 inhiben la producción de renina y dificultan la redistribución del potasio al espacio intracelular; la heparina inhibe la producción de aldosterona; y los glucósidos digitálicos bloquean la Na-K-ATPasa y, por tanto, perjudican la secreción de potasio del conducto colector ${ }^{(6)}$. (Figura 1).

Figura 1. Mecanismos de acción del desarrollo de hiperpotasemia en pacientes con enfermedad renal crónica y condiciones comórbidas asociadas.

AKI, lesión renal aguda; CKD, enfermedad renal crónica; CVD, enfermedad cardiovascular; DM, diabetes mellitus; GFR, tasa filtración glomerular; RAAS, Sistema renina-angiotensina aldosterona Kovesdy CP, Am J Med 128:1281-1287, 2015. ${ }^{(6)}$

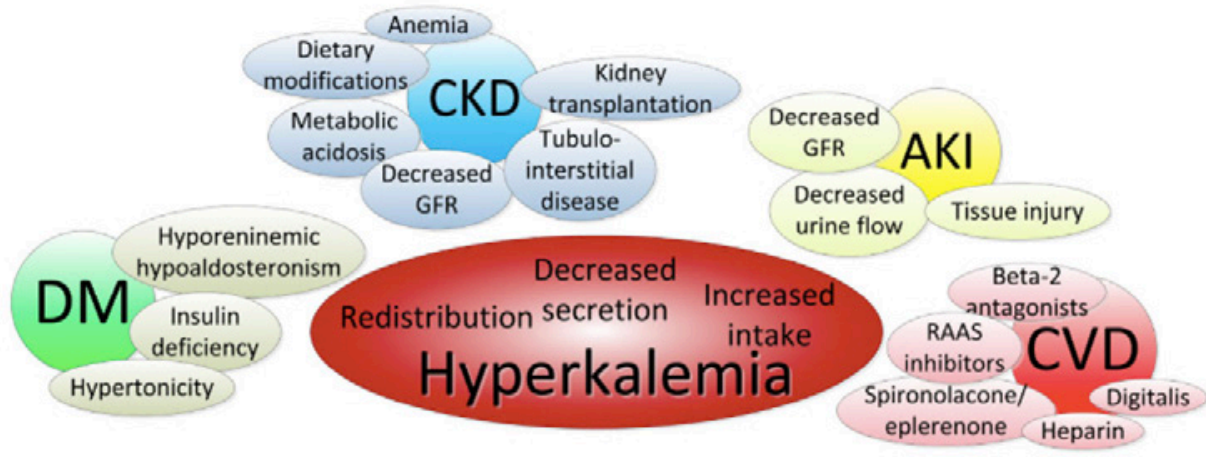

Los paradigmas actuales de abordaje de la hiperpotasemia crónica se centran en la eliminación de los factores de predisposición, como la ingesta elevada de potasio en la dieta o los suplementos, y el uso de medicamentos que se sabe que elevan el nivel de potasio. Entre estos últimos, los inhibidores del sistema renina angiotensina aldosterona (RAASi) son algunos de los medicamentos más utilizados, y su interrupción suele ser el primer paso que dan los médicos para prevenir la reaparición de la hiperpotasemia ${ }^{(2,7)}$. Si bien esta estrategia suele tener éxito, también priva a los pacientes de los beneficios reconocidos de esta clase, como sus efectos cardio y renoprotecto$r^{r e s}{ }^{(8)}$. La llegada de nuevas resinas de intercambio catiónico plantea un nuevo escenario clínico en el tratamiento de la hiperpotasemia crónica, al permitir el uso continuado de este grupo de medicamentos, beneficiándose el paciente de su efecto beneficioso a nivel cardiovascular. 
El Patiromero (Veltassa $\left.{ }^{\circledR}\right)$ es un polímero no absorbible que intercambia el potasio con el calcio, y que recientemente ha sido aprobado por la FDA para el tratamiento de la hiperpotasemia ${ }^{(1,2)}$. Diversos estudios han evidenciado la eficacia y seguridad de patiromer, en comparación con poliestireno sulfonato de sodio (Resinsodio $®$ ), en paciente con hiperpotasemia crónica y tratamiento concomitante con RAASi ${ }^{(9,10)}$. Patiromero es generalmente bien tolerado tanto de forma aguda como a largo plazo. Los síntomas adversos más comunes están relacionados con el sistema gastrointestinal, el estreñimiento o la hipomagnesemia ${ }^{(10)}$.

En este artículo se plantea, a tenor de un caso clínico, una revisión sobre el abordaje diagnóstico y terapéutico de la hiperpotasemia desde el servicio de urgencias hasta la unidad hospitalización a domicilio, haciendo especial hincapié en los nuevos agentes terapéuticos y los posibles beneficios de su aplicación.

\section{DESARROLLO DE LA EXPERIENCIA}

Informamos de un caso clínico de una paciente de 67 años, extranjera, que es traída por ambulancia tras sospecha de ICTUS. A su llegada a urgencias presenta imposibilidad de movilización de ambos miembros superiores acompañado de astenia y malestar general de 5 horas de evolución. Refería cuadro de vómitos autolimitados hace 2 días, sin alteraciones en el lenguaje, desviación de la comisura bucal u otra clínica sobreañadida. Como antecedentes personales a destacar presentaba una HTA controlada con amlodipino $10 \mathrm{mg}$, enalapril $10 \mathrm{mg}$ y metroprolol $25 \mathrm{mg}$, DM insulinodependiente y nefrectomia por cáncer renal en 2016. Ante hallazgos en exploración física y electrocardiograma (ECG) (figura 2), se plantea diagnóstico de hiperpotasemia administrándose bolo gluconato cálcico al 10\% con repetición del ECG a los 10min, objetivando normalización en complejo QRS. Se solicita analítica sanguínea y TAC abdomen, dónde se aprecia un filtrado glomerular $6 \mathrm{~mL} / \mathrm{min} / 1,73 \mathrm{~m} 2$, urea $149 \mathrm{mg} / \mathrm{dl}$, creatinina $7 \mathrm{mg} / \mathrm{dl}$, CK $115 \mathrm{mg} / \mathrm{dl}$, sodio $124 \mathrm{mEq} / \mathrm{L}$, potasio de $9.5 \mathrm{mEq} / \mathrm{L}$ y litiasis obstructiva en tercio proximal uréter izquierdo junto con uretero-hidronefrosis grado III (figura 3).

Figura 2. Se aprecian un aplanamiento de onda P. Ondas T picudas en V2-V6 y elevación segmento ST en derivaciones inferiores (II, III y aVF)

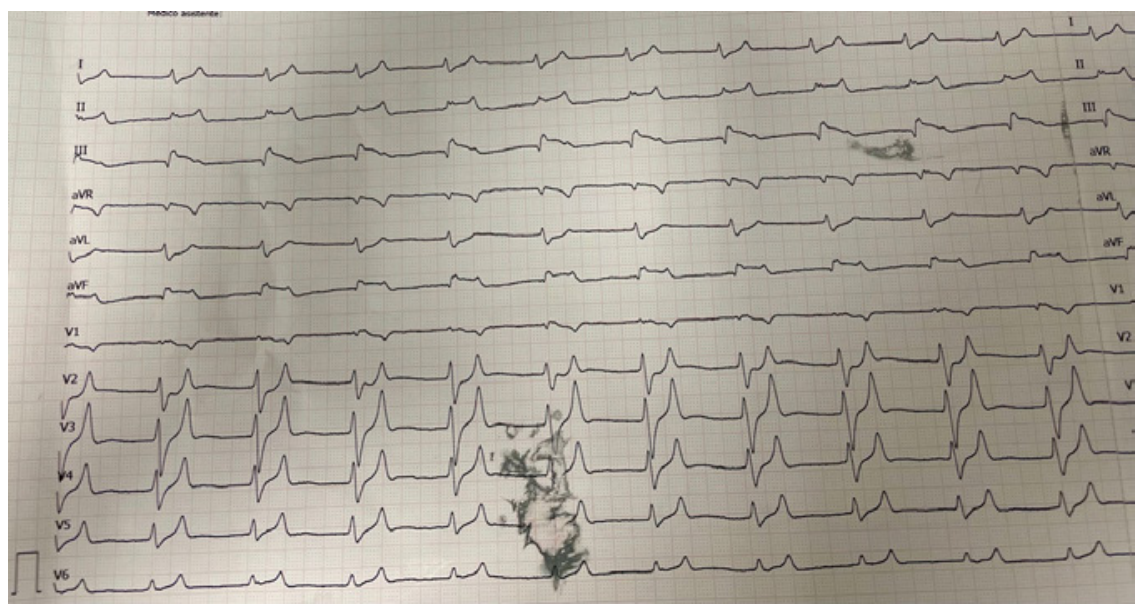


Figura 3. litiasis obstructiva en tercio proximal uréter izquierdo junto con uretero-hidronefrosis grado III

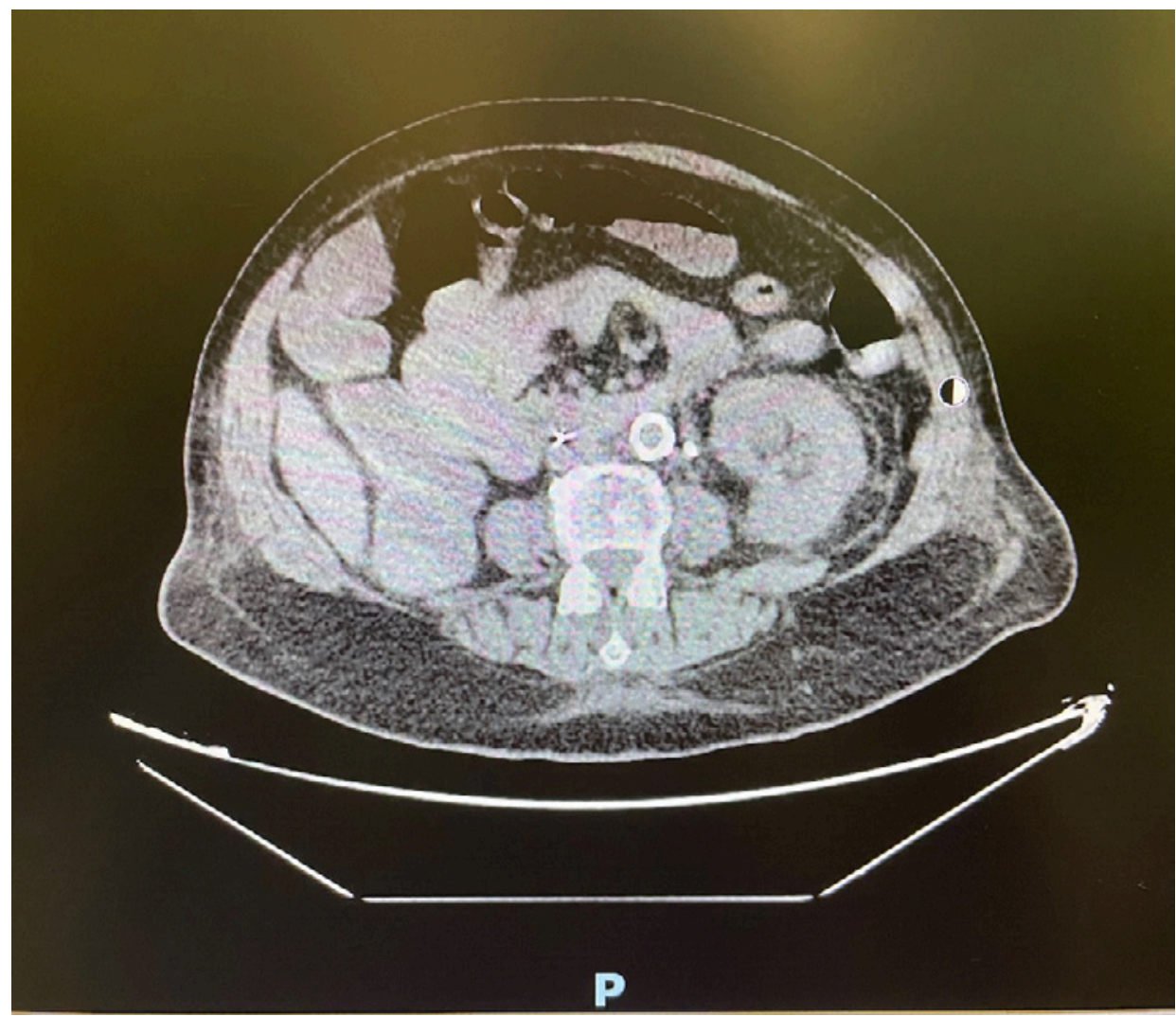

Durante el ingreso se realiza corrección de hiperpotasemia con insulina más glucosa al 50\% y agonista beta-2 según algoritmo(1). Se procede en quirófano a citoscopia con colocación de doble J izquierdo. Finalmente, es remitido por el servicio de medicina interna a Unidad de Hospitalización a Domicilio de San Juan de Alicante para tratamiento de hiperpotasemia. A la llegada a nuestra unidad, presenta un FG de $27 \mathrm{~mL} / \mathrm{min} / 1,73 \mathrm{~m} 2$, una creatinina de $3.7 \mathrm{mg} / \mathrm{dl}$ y potasio de $6,4 \mathrm{mEq} / \mathrm{L}$. Se decide iniciar tratamiento durante 5 días con furosemida $20 \mathrm{mg}$ iv y patiromero $8,4 \mathrm{~g}$ vía oral diario manteniendo enalapril 10mg durante este periodo, presentando la paciente cifras normotensionales y una correcta diuresis durante curso clínico. La valoración al $3^{\circ}$ día la paciente refirió un cuadro de flatulencia y diarrea autolimitada en 24 horas, sin repercusión en cuadro clínico. En el día 6 de inicio de tratamiento se realiza analítica en sangre con valores de $F G 41 \mathrm{~mL} / \mathrm{min} / 1,73 \mathrm{~m} 2$, creatinina de $2.6 \mathrm{mg} / \mathrm{dl}$, potasio de $5,1 \mathrm{mg} / \mathrm{dl}$ y calcio de $9.1 \mathrm{mg} / \mathrm{dl}$. Ante resultados analíticos, se decide retirada de furosemida y continuidad de tratamiento con IECA y patiromero cálcico. 


\section{DISCUSIÓN}

La optimización en el tratamiento de pacientes pruripatológicos es una de los objetivos de los cuidados paliativos. El aumento de la prevalencia de las ECV, la DM y la ERC, aunado al creciente uso de los RAASi, han aumentado la incidencia de la hiperpotasemia y la necesidad de actualización respecto a opciones terapéuticas eficaces y seguras en este grupo de pacientes. En el presente caso, la estrategia de tratamiento utilizada demostró ser altamente efectiva en un paciente con hiperpotasemia y ERC agudizada, no sólo en el control de síntomas y del potasio, sino también en la continuidad del tratamiento con RAASi, beneficiándose con ello la paciente de sus efectos reno y cardioprotectores.

Este caso sugiere que una combinación de tratamiento con fármacos RAASi, diuréticos de asa y resinas de intercambio catiónico (patiromero) es segura y presenta una mejoría en el control de la hiperpotasemia y sus síntomas asociadados.

\section{BIBLIOGRAFÍA}

1. Rafique Z, Chouihed T, Mebazaa A, Frank Peacock W. Current treatment and unmet needs of hyperkalaemia in the emergency department. Eur Heart J Suppl. 2019;21(Suppl A):A12-A19. DOI: 10.1093/eurheartj/suy029

2. Kovesdy CP. Updates in hyperkalemia: Outcomes and therapeutic strategies. Rev Endocr Metab Disord. 2017;18(1):41-47. DOI:10.1007/s11154-016-9384-x

3. Hayes J, Kalantar-Zadeh K, Lu JL, Turban S, Anderson JE, Kovesdy CP. Association of hypoand hyperkalemia with disease progression and mortality in males with chronic kidney disease: the role of race. Nephron Clin Pract. 2012;120(1):c8-16. DOI: 10.1159/000329511

4. Rossignol P, Legrand M, Kosiborod M, Hollenberg SM, Peacock WF, Emmett M, et al. Emergency management of severe hyperkalemia: Guideline for best practice and opportunities for the future. Pharmacol Res. 2016;113(Pt A):585-91. DOI: 10.1016/j.phrs.2016.09.039.5

5. Truhlář A, Deakin CD, Soar J, Khalifa GEA, Alfonzo A, Bierens JJLM, et al. European Resuscitation Council Guidelines for Resuscitation 2015: Section 4 - Cardiac arrest in special circumstances. Resuscitation. 2015;95:148-201. DOI: 10.1016/j.resuscitation.2015.07.017

6. Kovesdy CP. Management of Hyperkalemia: An Update for the Internist. Am J Med. 2015;128(12):1281-7. DOI: 10.1016/j.amjmed.2015.05.040

7. Kovesdy CP. Management of hyperkalaemia in chronic kidney disease. Nat Rev Nephrol. 2014;10(11):653-62. DOI: 10.1038/nrneph.2014.168

8. Marín R, Álvarez-Navascués R, Fernández-Vega F. Bloqueo del sistema renina-angiotensinaaldosterona en hipertensión arterial, diabetes y nefropatía. Rev Esp Cardiol Supl. 2008;8(E). DOI: 10.1016/S1131-3587(08)76104-1

9. Bakris GL, Pitt B, Weir MR, Freeman MW, Mayo MR, Garza D, et al. Effect of Patiromer on Serum Potassium Level in Patients With Hyperkalemia and Diabetic Kidney Disease: The AMETHYSTDN Randomized Clinical Trial. JAMA. 2015;314(2):151-61. DOI: 10.1001/jama.2015.7446

10. Weir MR, Bakris GL, Bushinsky DA, Mayo MR, Garza D, Stasiv Y, et al. Patiromer in patients with kidney disease and hyperkalemia receiving RAAS inhibitors. N Engl J Med. 2015;372(3):211-21. DOI: 10.1056/NEJMoa1410853 